\title{
Duração dos Episódios de Fibrilhação Auricular e Implicações no Risco Tromboembólico
}

\author{
Duration of Atrial Fibrillation Episodes and Implications for the \\ Thromboembolic Risk
}

\author{
David NEVES $\triangle 1$, Pedro Silva CUNHA ${ }^{2}$, Mário OLIVEIRA ${ }^{2}$ \\ Acta Med Port 2015 Nov-Dec;28(6):766-772
}

RESUMO

Introdução: A fibrilhação auricular é uma arritmia comum e com risco tromboembólico bem documentado, estando definidas nas recomendações internacionais indicações referentes ao uso de anticoagulantes orais. Existem, contudo, lacunas de informação nomeadamente no que se refere à duração dos episódios de fibrilhação auricular e sua relação com o risco de tromboembolismo. Esta questão tem particular interesse em doentes com dispositivos electrónicos cardíacos implantados com documentação contínua da duração de episódios de taquidisritmias auriculares, que são frequentemente curtos e assintomáticos.

Material e Métodos: Foi feita uma análise crítica da evidência disponível sobre a relação da duração dos episódios de FA paroxística e a ocorrência de eventos embólicos, com base numa pesquisa na base de dados bibliográfica PubMed.

Resultados: Foram selecionados oito artigos com abordagens diferentes no estudo deste tema; sete com recurso a monitorização cardíaca com dispositivos electrónicos cardíacos implantados (pacemakers, cardioversores-desfibilhadores implantáveis e ressincronizadores cardíacos) e um com base em registo de Holter. Metade destas publicações, correspondendo globalmente às maiores amostragens, aborda a questão do ponto de vista do somatório diário de episódios de fibrilhação auricular (carga diária) e não da duração de cada episódio. O risco tromboembólico aumenta gradualmente com a carga arrítmica, tendo sido demonstrado um aumento significativo do risco quando esta ultrapassa os cinco minutos num dia.

Discussão: A formação de um trombo intracavitário, e consequente potencial embólico, é um processo dinâmico que resulta da interacção de várias condicionantes anatómicas e funcionais. O risco individual dependerá da interacção destes factores. A associação entre fenómenos embólicos e curtos períodos de fibrilhação auricular é inequívoca, apesar do mecanismo não ser óbvio, tendo em conta a discrepância frequentemente observada entre os períodos de fibrilhação auricular e os eventos clínicos.

Conclusões: O risco de eventos tromboembólicos aumenta significativamente mesmo para períodos curtos de fibrilhação auricular ( $\geq$ cinco minutos de fibrilhação auricular em um dia), apesar da relação causa-efeito não estar definida. A decisão final sobre o recurso à anticoagulação oral deve basear-se na avaliação clínica individualizada.

Palavras-chave: Fibrilhação Auricular; Tromboembolia/etiologia.

\section{ABSTRACT}

Introduction: Atrial fibrillation is the most common chronic arrhythmia in clinical practice, which is associated with a well known increased thromboembolic risk. The use of oral anticoagulants in this context is well established. However, there are some gaps in information that warrant further studies, such as the duration of an atrial fibrillation event that is long enough to increase the risk of embolic phenomena. This may be of important clinical concern, particularly in patients with cardiac implanted devices, in which very short periods of asymptomatic atrial fibrillation are often detected.

Material and Methods: We performed a critical review on the association of brief atrial fibrillation episodes and thromboembolic events, based on available literature indexed on PubMed.

Results: After initial selection of abstracts and checking of references a final pool of 8 papers were analysed; seven describing studies with cardiac implanted devices and one with Holter monitoring. Four of the studies addressed this issue with a 'daily burden' approach rather than single episode duration. The risk increases with the magnitude of atrial fibrillation burden, with 5 minutes of atrial fibrillation in one day being the shortest time shown to independently predict thromboembolic events.

Discussion: The formation of an intracardiac thrombus, and respective embolic potential, is a dynamic process resulting from the interaction of anatomical and functional variables. The individual risk will depend on these factors. The association between embolic events and short atrial fibrillation episodes is evident, although the mechanism is not obvious, given the time discrepancy that is frequently observed between atrial fibrillation episode and clinical event.

Conclusions: An atrial fibrillation burden of 5 minutes in one day has been shown to be independently associated with a significantly increased risk, although the cause-effect mechanism is not clear. A standardized way to select patients with short-duration atrial fibrillation periods that will have a meaningful benefit of chronic oral anticoagulation is still to define. Therefore, decisions should be made in an individualized manner.

Keywords: Atrial Fibrillation; Thromboembolism/etiology.

\section{INTRODUÇÃO}

A fibrilhação auricular (FA) é a arritmia mais comum na prática clinica, ${ }^{1}$ com um risco tromboembólico acrescido bem documentado, ${ }^{2}$ e que tem vindo a justificar indicações terapêuticas bem definidas nas recomendações internacionais. ${ }^{2,3}$ As actuais recomendações defendem uma abordagem cada vez mais interventiva no sentido da prevenção

\footnotetext{
1. Serviço de Cardiologia. Hospital do Espírito Santo. Évora. Portugal.

2. Serviço de Cardiologia. Hospital de Santa Marta. Centro Hospitalar Lisboa Central. Lisboa. Portugal.

$\triangle$ Autor correspondente: David Neves. dcneves25@hotmail.com

Recebido: 02 de Março de 2015 - Aceite: 12 de Outubro de 2015 | Copyright @ Ordem dos Médicos 2015
} 
de eventos embólicos com anticoagulação oral, sugerindo o uso desta medicação em todos os doentes, desde que aceitável do ponto de vista hemorrágico, excepto nos de verdadeiro baixo risco, definido casos como doentes com um score $\mathrm{CHA}_{2} \mathrm{DS}_{2}-$ VASc $=0$.

Uma situação frequente no seguimento de doentes com dispositivos electrónicos cardíacos implantados (DECl), como pacemakers, cardioversores-desfibrilhadores, sistemas de ressincronização cardíaca e registadores de eventos, é a detecção de períodos de FA, frequentemente assintomáticos. Na realidade, estes dispositivos abrem uma janela de oportunidade de estudo desta arritmia no que respeita à sua incidência, duração e relação com o risco tromboembólico associado, no sentido de melhor estratificar e tratar os doentes. No entanto, permanece muitas vezes a dúvida na decisão terapêutica de anticoagular ou não, tendo em conta que muitos dos episódios são auto-limitados e de curta duração (Fig. 1). ${ }^{4}$ Tendo em conta esta questão clinica recorrente, foi feita uma revisão bibliográfica deste tema, com base na literatura recente.

\section{MATERIAL E MÉTODOS}

Foi feita uma pesquisa inicial na base de dados bibliográfica PubMed (http://www.ncbi.nlm.nih.gov/pubmed) utilizando como palavras-chave 'atrial fibrillation - duration risk stroke'. Foram pré-seleccionados os artigos potencialmente dirigidos a esta temática através da leitura dos seus resumos. Após análise da versão integral dos trabalhos relevantes, foram analisadas também as suas referências bibliográficas. Posteriormente feita uma selecção dos trabalhos mais pertinentes com base nos seguintes critérios: estudo com mais de 200 doentes, seguimento clínico de pelo menos um ano, com avaliação da mortalidade e/ou fenómenos tromboembólicos.

\section{RESULTADOS}

Foram seleccionados oito estudos cujos resultados se discutem por ordem cronológica de publicação e cuja informação essencial se sumariza na Tabela 1 . Nestas pu- blicações, a maior evidência do risco tromboembólico está relacionada com a designação de 'carga de FA', que traduz o somatório de todos os períodos de tempo que o doente está em FA durante um dia (daily AF burden).

$\mathrm{O}$ artigo de Glotzer et $\mathrm{al}^{5}$ consiste na análise dos dados dum subgrupo do estudo Mode Selection Trial (MOST), um estudo randomizado com pacemakers DDDR versus VVIR em 2010 doentes com doença do nódulo sinusal. Os dispositivos foram programados para registar episódios de elevada frequência auricular (> $220 \mathrm{bpm}$ ) com pelo menos 10 batimentos consecutivos. Foi demonstrado um aumento de mortalidade e de acidente vascular cerebral (AVC) isquémico em indivíduos com episódios de duração superior a cinco minutos.

O trabalho de Capucci et $\mathrm{al}^{6}$ foi realizado em doentes com bradicardia e documentação prévia de FA paroxística, submetidos a implantação de pacemakers com capacidade de terapêutica antitaquicardia, tendo demonstrado que períodos de FA superiores a 24 horas são preditores independentes de eventos embólicos. O TRENDS ${ }^{7}$ foi um estudo prospectivo, observacional, que avaliou o risco tromboembólico de episódios de taquicardia/fibrilhação auricular em doentes com $\mathrm{DECl}$ e pelo menos um factor de risco de AVC. Os dispositivos eram interrogados de três em três meses e foi considerado o dia com maior carga de FA nos últimos 30 dias. Demonstrou-se que doentes com pelo menos um dia com $\geq 5,5$ horas de FA nos últimos 30 dias têm o risco de fenómenos tromboembólicos aumentado para cerca do dobro. No trabalho de Botto et $\mathrm{al}^{8}$ é feita uma abordagem diferente, demonstrando-se uma melhoria da capacidade preditora de eventos do score de risco $\mathrm{CHADS}_{2}$ quando combinada com a carga diária de FA. Foram seguidos ao longo de um ano portadores de pacemaker com capacidade de detecção e registo de taquidisritmias auriculares e, com base nos eventos tromboembólicos registados, foram identificados dois grupos com risco distinto $(0,8 \%$ vs $5 \%$, $p=0,035)$, sendo de baixo risco os doentes sem FA com $\mathrm{CHADS}_{2} \leq 2$, ou com carga de FA $\geq 5$ minutos em $\geq 1$ dia no ano com $\mathrm{CHADS}_{2} \leq 1$, ou $\geq 1$ período de $\mathrm{FA} \geq 24$ horas

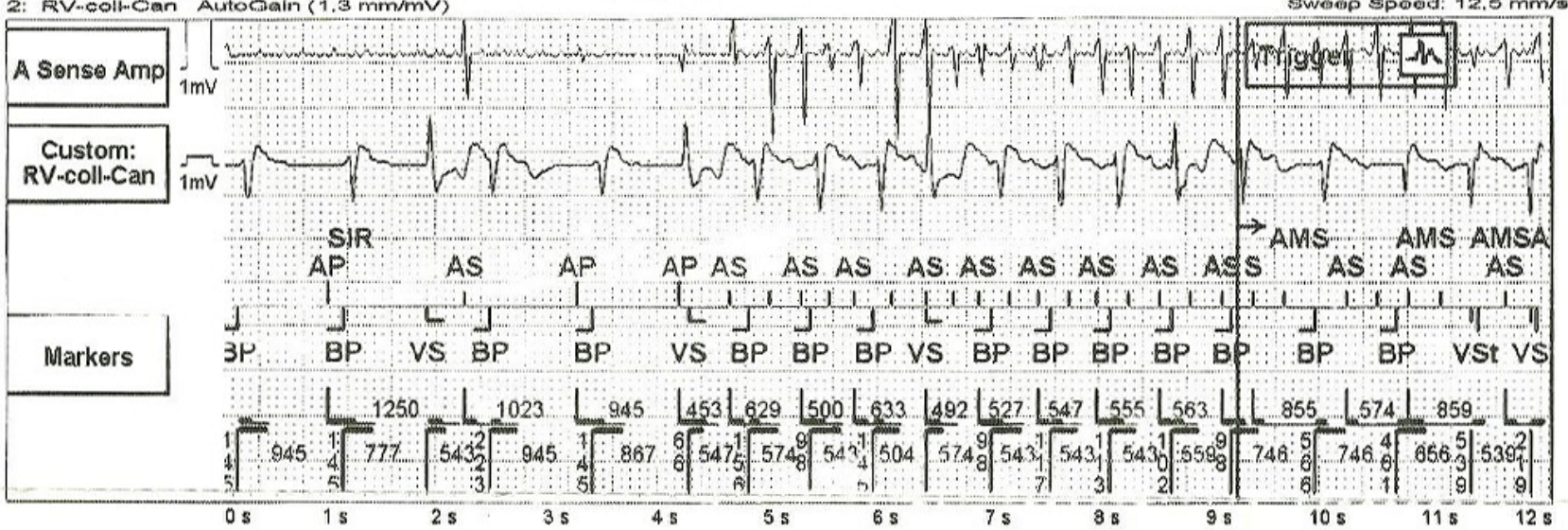

Figura 1 - Exemplo de um episódio de fibrilhação auricular com 38 seg de duração detectado casualmente numa consulta de seguimento de um doente com dispositivo de ressincronização cardíaca 


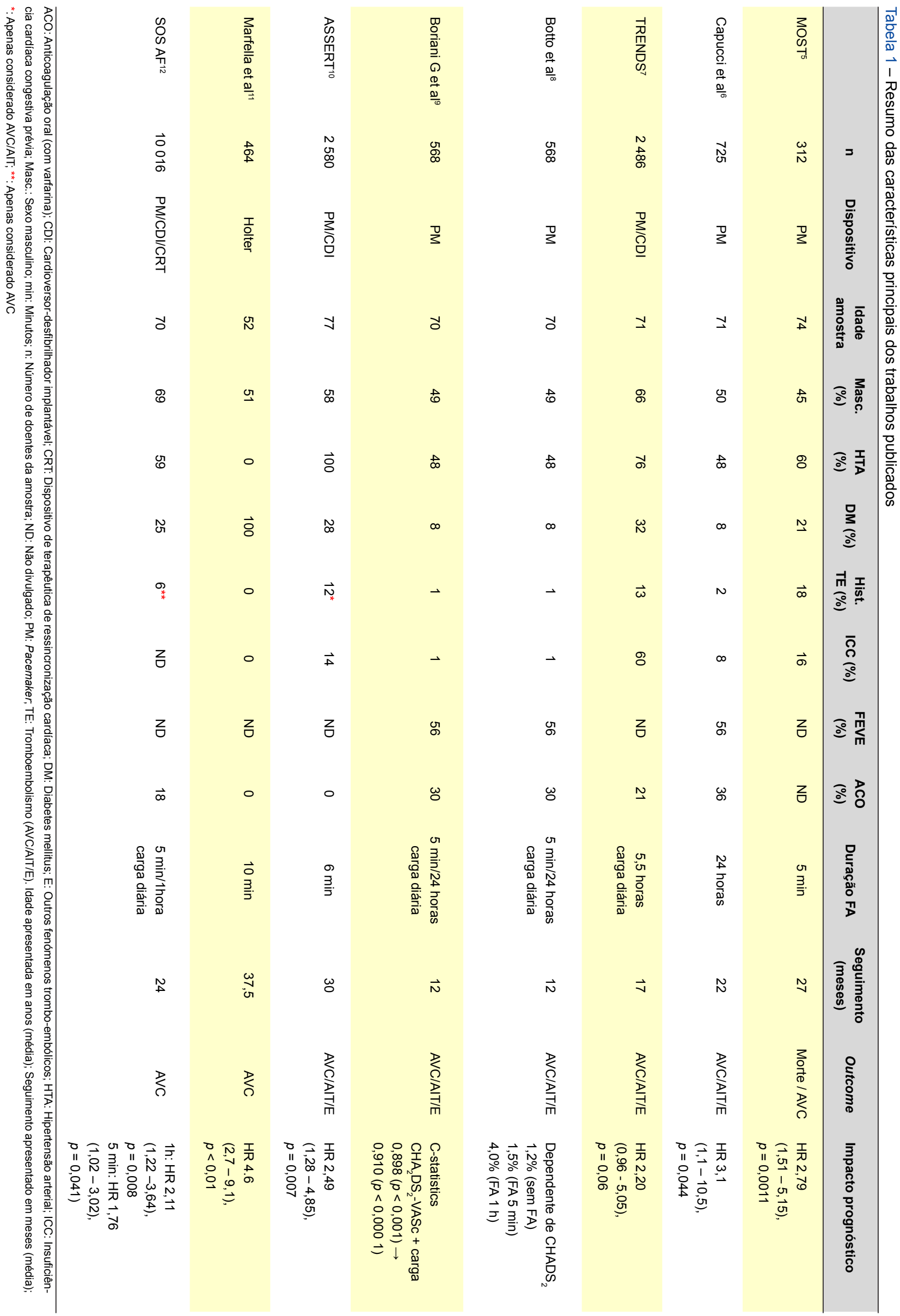


com $\mathrm{CHADS}_{2}=0.0$ mesmo grupo ${ }^{9}$ demonstrou posteriormente, e com a mesma amostra de doentes, a capacidade da carga de FA melhorar a capacidade preditora de risco de tromboembólico, mas agora com o score $\mathrm{CHA}_{2} \mathrm{DS}_{2}$-VASc. Novamente, os valores de carga de FA de $\geq 5$ minutos em $\geq$ 1 dia, e $\geq 1$ período de 24 horas foram usados, permitindo um maior refinamento deste score. $\mathrm{O}$ critério de $\mathrm{CHA}_{2} \mathrm{DS}_{2}$. -VASc $\geq 3$ ou concomitantemente $\mathrm{CHA}_{2} \mathrm{DS}_{2}-\mathrm{VASc}=2 \mathrm{e}$ carga de $F A \geq 24$ horas permite um aumento da especificidade sem prejuízo significativo da sensibilidade (42 e 93\%, respectivamente) em comparação com o critério clássico $\mathrm{CHA}_{2} \mathrm{DS}_{2}-\mathrm{VASc} \geq 1$ (7 e 100\%. respectivamente), melhorando $C$-statistics de 0,898 para $0,910(p<0,0001)$.

O estudo ASSERT ${ }^{10}$ foi o maior estudo encontrado analisando o risco tromboembólico em relação à duração dos períodos de FA individualmente. De forma resumida, este estudo envolveu 2580 doentes com pacemakers ou cardiodesfibrilhadores implantáveis (CDI), em quem foram registados episódios de actividade auricular de elevada frequência (> 190 bpm) com duração superior a seis minutos durante um período de monitorização de três meses. Demonstrou-se que, num seguimento clínico de 2,5 anos, a detecção destes períodos se associou independentemente a maior incidência de AVC e embolia sistémica. Outro grupo, de Marfella et al, ${ }^{11}$ com uma população de doentes diabéticos submetidos a monitorização com registo de Holter de 48 horas de três em três meses, mostrou que períodos de FA superiores a 10 minutos são preditores independentes de AVC e AVC assintomático (diagnosticado com ressonância magnética nuclear). Finalmente, o projecto SOS $A F,^{12}$ o mais recente trabalho publicado e mais significativo a nível de amostragem, contemplando um total de 10016 doentes (conjunto de doentes de três estudos: TRENDS, ${ }^{7}$ PANORAMA e Italian Clinical Service Registry Project) com DECl. Num seguimento clínico a dois anos, $43 \%$ dos indivíduos tiveram pelo menos um dia com cinco minutos de carga de FA, o que se associou a um aumento de AVC isquémico independentemente do score $\mathrm{CHADS}_{2} \mathrm{e}$ da medicação com anticoagulantes. A mediana de tempo de seguimento necessário nestes doentes para que se verificasse um dia com carga $\geq 5$ minutos foi de dois meses. Foram avaliados os efeitos clínicos de diferentes valores de carga diária máxima. A presença de pelo menos um dia com uma carga de $\geq 1$ hora apresentou-se como o mais significativo, associando-se a uma duplicação do risco de AVC isquémico (HR 2,11; IC 95\%: 1,22 - 3,64, $p=0,008$ ).

\section{DISCUSSÃO}

Apesar de várias limitações e condicionantes, tem sido mostrado em diferentes estudos que o risco tromboembólico aumenta de forma significativa para cargas de FA superiores a 5 minutos num dia, mesmo para episódios assintomáticos, ${ }^{12}$ o que constitui um dado clínico de grande relevância, particularmente numa altura em que o recurso a novos anticoagulantes torna o seguimento a longo prazo mais facilitado, com um perfil de segurança melhorado e com bons níveis de eficácia.
Do ponto de vista fisiopatológico é importante notar que a formação de um trombo auricular esquerdo, em contexto de FA (regra geral no apêndice auricular esquerdo), pode ser interpretado com analogia à tríade de Virchow,,$^{13}$ habitualmente aplicada à trombose venosa. Esta contempla três vertentes em interacção: fluxo do sangue, superfície celular e coagulabilidade do sangue. A influência do fluxo na formação do trombo é determinante, estando a coagulabilidade tão aumentada quanto maior a estase. São vários os mecanismos pelos quais a FA diminui a contractilidade auricular, como sejam as alterações do metabolismo do $\mathrm{Ca}^{++}$intracelular e da fosforilação dos miofilamentos. Esta contractilidade pode demorar dias ou semanas a recuperar após a restauração do ritmo sinusal, sendo por isso muito importante a manutenção da anticoagulação durante este período. ${ }^{14}$ As condições da dinâmica de fluxos na aurícula esquerda, particularmente no que se refere ao apêndice auricular, vão depender não só da sua contractilidade deste, como também do seu formato e área ostial. ${ }^{15,16}$ Existe também evidência de disfunção endotelial na FA, com diminuição da produção de óxido nítrico e dos fatores antitrombóticos e com aumento de fatores protrombóticos. ${ }^{13}$ A influência da coagulabilidade do sangue é determinada não só por variadas condições bioquímicas não modificáveis, como variantes genéticas, mas também alterações da hemostase associadas à $\mathrm{FA}$, que cursam com aumento da reciclagem da fibrina. ${ }^{17}$

É interessante verificar que apesar da forte associação estatística entre curtos períodos de FA e eventos tromboembólicos, não é clara a sua relação de causalidade. Por exemplo, numa recente análise do estudo ASSERT procurando a relação temporal entre a detecção do período de FA e a ocorrência de AVC ou embolia sistémica, ${ }^{18}$ dos 51 doentes com este evento clínico apenas quatro (8\%) tiveram um período de FA nos 30 dias prévios. Este fenómeno pode ter várias explicações, nomeadamente a possibilidade de nestes doentes a FA ser um importante marcador do risco de AVC, associado a fragilidade cardiovascular global, não sendo obrigatoriamente o mecanismo fisiopatológico pelo qual o AVC acontece. Não se pode excluir a possibilidade de episódios de $F A<6$ minutos serem suficientes para a formação de um trombo ${ }^{19} \mathrm{e}$, neste contexto, está descrita a associação de episódios de taquicardia auricular com $\geq 20$ batimentos consecutivos ou $\geq 30$ extra-sístoles supra-ventriculares por hora com ocorrência de AVC..$^{20,21}$ No entanto uma futura sub-análise da amostra do estudo ASSERT com períodos de menor duração não será possível, já que o protocolo deste estudo contemplou por definição apenas registo de episódios superiores a seis minutos. ${ }^{22}$ Contudo, os autores não consideram isto uma limitação, já que dos episódios de $<6$ minutos que foram eventualmente analisados, <50\% correspondiam a FA. Numa sub-análise do estudo TRENDS ${ }^{23}$ já se tinham documentado achados sobreponíveis, com apenas $50 \%$ dos doentes com eventos a terem a arritmia documentada previamente e apenas 55\% destes com a arritmia nos últimos 30 dias antes do evento. A hipótese dos fenómenos de curtos períodos de FA 
constituírem um marcador de risco, e não obrigatoriamente um mecanismo tromboembólico, é corroborado pelos achados recentes de Gonzalez et al, ${ }^{24}$ que demonstraram que períodos de frequência auricular rápida ( $\geq 178 \pm 12$ bpm durante $\geq 5 \mathrm{~min}$ ) são preditores independentes de mortalidade cardiovascular. FA com início pós-operatório em doentes submetidos a cirurgia de revascularização do miocárdio demonstrou também ser um preditor independente de mortalidade a longo prazo. ${ }^{25}$

A utilidade da decisão terapêutica baseada na monitorização contínua com $\mathrm{DECl}$ foi recentemente testada no estudo IMPACT ${ }^{26}$ Neste estudo prospectivo, 2718 doentes foram randomizados para terapêutica convencional versus anticoagulação baseada na detecção de FA em monitorização remota. Foi definida taquicardia auricular como frequência auricular $\geq 300$ ciclos/min ( $\geq 36$ em 48 ciclos consecutivos). O protocolo de decisão de anticoagulação era complexo, tomando em consideração a carga arrítmica e o risco tromboembólico determinado pelo score $\mathrm{CHADS}_{2}$, podendo a terapêutica anticoagulante ser terminada na ausência de arritmia durante 30 ou 90 dias (conforme CHADS $_{2} 1$ - 2 ou 3 - 4, respetivamente). Os antagonistas da vitamina $\mathrm{K}$ foram a opção de anticoagulação mais utilizada. Os novos anticoagulantes eram permitidos, tendo começado a ser usados aquando da sua aprovação legal. $O$ estudo foi terminado precocemente por ausência de resultados estatisticamente significativos. Houve uma tendência não significativa para redução do AVC isquémico (HR 0,79 $[0,45$ - 1,39]) e aumento de hemorragias major ( $\operatorname{HR} 1,39$ $[0,89$ - 2,17]) no grupo de intervenção, com efeito neutro no endpoint primário (composto de AVC + embolia sistémica + hemorragia major). Este estudo não permite, contudo, uma afirmação definitiva acerca do manejo clínico destes doentes, ${ }^{27}$ devido a várias limitações e questões polémicas, como a suspensão da terapêutica.

A metodologia baseada na 'carga de FA' merece alguma atenção, sendo discutível se muitos episódios de curta duração terão a mesma trombogeneicidade que menos episódios mais longos, ainda que confiram a mesma carga de FA. A definição de FA/taquicardia auricular não é uniforme entre os estudos e provavelmente são detectados não só verdadeiros episódios de FA mas também episódios de flutter e taquicardia auricular, situações que, ao provocar diferentes alterações da contractilidade auricular e, consequentemente, do fluxo sanguíneo no seu interior, terão diferente impacto na trombogeneicidade. Não há geralmente nestes estudos informações anatómicas importantes, como o tamanho da aurícula esquerda, com potencial impacto na duração necessária da FA para provocar a formação de um trombo.

Do ponto de vista teórico, numa situação em que um doente esteja num estado protrombótico (polimorfismos genéticos procoagulantes, inflamação ou outros), tenha uma aurícula esquerda francamente dilatada, com pouca contractilidade e com lesão endotelial, é possível que seja necessário menos tempo em FA para que se forme um trombo com potencial para embolismo clinicamente relevante.
Nos testes mais habituais de coagulação são necessários apenas alguns segundos até se observar a formação de um trombo, mas sendo feito em condições artificiais é difícil extrapolar valores temporais para a formação de um trombo in-vivo. Poucos minutos podem, em determinadas situações, ser suficientes para a formação de um trombo, o que sublinha a relevância de curtos períodos de estagnação sanguínea. ${ }^{19}$

Classicamente o valor temporal de 48 horas tem sido aceite para nos indicar a necessidade de anticoagulação ou realização de ecocardiograma transesofágico para exclusão de trombos antes da realização de uma tentativa de conversão da FA, assumindo-se as 48 horas como um valor-limiar para o qual o risco tromboembólico passa a estar significativamente aumentado. ${ }^{2}$ No entanto, a presente revisão da literatura mostra que existe já bastante evidência de que o risco está aumentado mesmo com períodos de FA de duração bastante inferior.

Para definir com maior acuidade o risco tromboembólico de determinado indivíduo com episódios paroxísticos de FA seria necessário ter em conta todos os aspectos mencionados, desde características bioquímicas e genéticas da hemostase, a aspectos anatómicos e funcionais da aurícula e apêndice auricular esquerdos, para além da avaliação do risco global do doente, que já é contemplado, nomeadamente com o score $\mathrm{CHA}_{2} \mathrm{DS}_{2}$-Vasc. A criação de scores mais complexos que considerem todos estes factores pode parecer uma alternativa mais precisa, mas seria igualmente mais complexa e, consequentemente, menos prática.

Do ponto de vista prático, a atitude terapêutica mais importante para estes doentes é a anticoagulação oral. Numa altura em que os 'novos' anticoagulantes, antagonistas diretos da trombina e factor $X$ ativado, estão a ser cada vez mais usados, com maior segurança e eficácia quando comparados aos antigos antagonistas da vitamina $\mathrm{K}$, torna-se ainda mais pertinente definir critérios para diferenciar os doentes que beneficiam desta terapêutica. Será importante também conhecer melhor a causa dos eventos nesta população. Na possibilidade de a causa principal não ser cardioembólica, sendo FA apenas um marcador de risco, provavelmente haverá outras armas terapêuticas a investigar nesta população, de acordo com as recomendações internacionais para a prevenção do AVC. ${ }^{28,29}$

Os vários estudos apresentados têm amostras com diferentes proporções de doentes sob medicação com anticoagulantes, tanto no braço com FA documentada como no braço de doentes sem FA. A estatística poderia auxiliar a contornar esta situação numa eventual meta-análise, mas a robustez da evidência nunca será tão sólida como num estudo prospectivo e controlado. Nestes estudos, a anticoagulação foi feita com antagonistas da vitamina $\mathrm{K}$, o que pode ter influenciado os resultados clínicos, se tivermos em conta o potencial para maior segurança e eficácia dos novos anticoagulantes na população com FA não valvular. ${ }^{30-33}$

Não existem ainda estudos prospectivos, randomizados, que demonstrem a superioridade da anticoagulação em doentes com FA assintomática de curtos períodos 
detectada com dispositivos cardíacos implantáveis, pelo que a decisão deverá ser feita caso a caso, ponderando riscos e benefícios desta medicação. No entanto, a detecção de FA deve em todos os casos despoletar pelo menos a ponderação de prevenção com anticoagulação, independentemente da duração dos episódios. ${ }^{4}$

Vários estudos estão em curso e poderão aumentar o conhecimento nesta área, nomeadamente o ARTESiA (NCT 01938248), que randomiza doentes com FA detectada por dispositivos a medicação com apixabano versus aspirina, e o REACT COM (NCT 01706146), que avalia doentes com FA conhecida, e $\mathrm{CHADS}_{2}$ de 1 ou 2 monitorizados com registador de eventos implantável.

\section{Limitações do trabalho}

É importante referir que este trabalho se trata de uma revisão não sistemática de um tema que envolve conceitos com várias definições possíveis (i.e. período de FA, curta duração, eventos tromboembólicos), e que expõe evidência obtida em estudos com populações relativamente heterogéneas e com metodologias distintas.

Não se pretende com este trabalho argumentar a favor de algum tipo de prática clínica padronizada, mas antes rever um tema, apresentando informação relevante que suporte a decisão clínica em casos semelhantes, e fomentar futura investigação nesta área.

\section{CONCLUSÕES}

Tendo em conta a evidência disponível, a presença de períodos de FA que confiram uma carga arrítmica que atinja $\geq 5$ minutos em um dia associa-se a um risco tromboembólico aumentado, sendo o risco tanto maior quanto maior for a carga de FA. Se estes doentes beneficiam de terapêutica crónica com anticoagulantes orais é uma questão que não está ainda esclarecida, mas esta é uma opção terapêutica a considerar nesta população, sobretudo se na presença de alto risco tromboembólico e baixo risco hemorrágico. A relação de causalidade entre os curtos períodos de FA e os eventos clínicos não está estabelecida e estudos prospectivos de grande dimensão serão necessários para melhor estratificar a orientação terapêutica nestes doentes.

\section{CONFLITOS DE INTERESSE}

Os autores declaram não ter qualquer conflito de interesse relativamente ao presente artigo.

\section{FONTES DE FINANCIAMENTO}

Este trabalho não recebeu qualquer contribuição e subsídio ou bolsa.

\section{REFERÊNCIAS}

1. Bonhorst D, Mendes M, Adragão P, De Sousa J, Primo J, Leiria E, et al. Prevalence of atrial fibrillation in the Portuguese population aged 40 and over: the FAMA study. Rev Port Cardiol. 2010;29:331-50.

2. Camm AJ, Kirchhof P, Lip GYH, Schotten U, Savelieva I, Ernst S, et al. Guidelines for the management of atrial fibrillation: the Task Force for the Management of Atrial Fibrillation of the European Society of Cardiology (ESC). Eur Heart J. 2010;31:2369-429.

3. Singer DE, Albers GW, Dalen JE, Fang MC, Go AS, Halperin JL, et al. Antithrombotic therapy in atrial fibrillation: American College of Chest Physicians evidence-based clinical practice guidelines ( $8^{\text {th }}$ edition). Chest. 2008;133:546S-92.

4. Camm AJ. The role of continuous monitoring in atrial fibrillation management. Arrhythmia Electrophysiol Rev. 2014;3:48-50.

5. Glotzer TV, Hellkamp AS, Zimmerman J, Sweeney MO, Yee R, Marinchak $\mathrm{R}$, et al. Atrial high rate episodes detected by pacemaker diagnostics predict death and stroke: Report of the atrial diagnostics ancillary study of the MOde Selection Trial (MOST). Circulation. 2003;107:1614-9.

6. Capucci A, Santini M, Padeletti L, Gulizia M, Botto G, Boriani G, et al. Monitored atrial fibrillation duration predicts arterial embolic events in patients suffering from bradycardia and atrial fibrillation implanted with antitachycardia pacemakers. J Am Coll Cardiol. 2005;46:1913-20.

7. Glotzer TV, Daoud EG, Wyse DG, Singer DE, Ezekowitz MD, Hilker C, et al. The relationship between daily atrial tachyarrhythmia burden from implantable device diagnostics and stroke risk the trends study. Circ Arrhythmia Electrophysiol. 2009;2:474-80.

8. Botto GL, Padeletti L, Santini M, Capucci A, Gulizia M, Zolezzi F, et al. Presence and duration of atrial fibrillation detected by continuous monitoring: crucial implications for the risk of thromboembolic events. J Cardiovasc Electrophysiol. 2009;20:241-8.

9. Boriani G, Botto GL, Padeletti L, Santini M, Capucci A, Gulizia M, et al. Improving stroke risk stratification using the $\mathrm{CHADS}_{2}$ and $\mathrm{CHA}_{2} \mathrm{DS}_{2}-\mathrm{VASc}$ risk scores in patients with paroxysmal atrial fibrillation by continuous arrhythmia burden monitoring. Stroke. 2011;42:1768-70.

10. Healey JS, Connolly SJ, Gold MR, Israel CW, Van Gelder IC, Capucci A, et al. Subclinical atrial fibrillation and the risk of stroke. N Engl J Med. 2012;366:120-9.

11. Marfella R, Sasso FC, Siniscalchi M, Cirillo M, Paolisso P, Sardu C, et al. Brief episodes of silent atrial fibrillation predict clinical vascular brain disease in type 2 diabetic patients. J Am Coll Cardiol. 2013;62:525-30.
12. Boriani G, Glotzer TV, Santini M, West TM, De Melis M, Sepsi M, et al Device-detected atrial fibrillation and risk for stroke: An analysis of > 10000 patients from the SOS AF project (Stroke preventiOn Strategies based on Atrial Fibrillation information from implanted devices). Eur Heart J. 2014;35:508-16.

13. Iwasaki YK, Nishida K, Kato T, Nattel S. Atrial fibrillation pathophysiology: implications for management. Circulation. 2011;124:2264-74.

14. Nattel S, Opie LH. Controversies in atrial fibrillation. Lancet. 2006;367:262-72.

15. Lee JM, Shim J, Uhm JS, Kim YJ, Lee HJ, Pak HN, et al. Impact of increased orifice size and decreased flow velocity of left atrial appendage on stroke in nonvalvular atrial fibrillation. Am J Cardiol. 2014;113:963-9.

16. Di Biase L, Santangeli $P$, Anselmino M, Mohanty P, Salvetti I, Gili S, et al. Does the left atrial appendage morphology correlate with the risk of stroke in patients with atrial fibrillation? Results from a multicenter study. J Am Coll Cardiol. 2012;60:531-8.

17. Watson T, Shantsila E, Lip GY. Mechanisms of thrombogenesis in atrial fibrillation: Virchow's triad revisited. Lancet. 2009;373:155-66.

18. Brambatti M, Connolly SJ, Gold MR, Morillo CA, Capucci A, Muto C, et al. Temporal relationship between subclinical atrial fibrillation and embolic events. Circulation. 2014;129:2094-9.

19. Haft Jl. Letter by Haft regarding article, 'Temporal relationship between subclinical atrial fibrillation and embolic events'. Circulation. 2015;131:e336.

20. Binici Z, Intzilakis T, Nielsen OW, Køber L, Sajadieh A. Excessive supraventricular ectopic activity and increased risk of atrial fibrillation and stroke. Circulation. 2010;121:1904-11.

21. Larsen BS, Kumarathurai $P$, Falkenberg J, Nielsen OW, Sajadieh $A$. Excessive atrial ectopy and short atrial runs increase the risk of stroke beyond incident atrial fibrillation. J Am Coll Cardiol. 2015;66:232-41.

22. Healey JS, Brambatti M, Gold MR, Morillo CA, Capucci A, Muto C, et al. Response to letter regarding article 'Temporal relationship between subclinical atrial fibrillation and embolic events'. Circulation. 2015;131:e337-8.

23. Daoud EG, Glotzer TV, Wyse DG, Ezekowitz MD, Hilker C, Koehler J, et al. Temporal relationship of atrial tachyarrhythmias, cerebrovascular events, and systemic emboli based on stored device data: a subgroup analysis of TRENDS. Heart Rhythm. 2011;8:1416-23.

24. Gonzalez M, Keating RJ, Markowitz SM, Liu CF, Thomas G, Ip JE, et 
al. Newly detected atrial high rate episodes predict long-term mortality outcomes in patients with permanent pacemakers. Heart Rhythm. 2014;11:2214-21.

25. El-Chami MF, Kilgo P, Thourani V, Lattouf OM, Delurgio DB, Guyton $\mathrm{RA}$, et al. New-onset atrial fibrillation predicts long-term mortality after coronary artery bypass graft. J Am Coll Cardiol. 2010;55:1370-6.

26. Martin DT, Bersohn MM, Waldo AL, Wathen MS, Choucair WK, Lip GY, et al. Randomized trial of atrial arrhythmia monitoring to guide anticoagulation in patients with implanted defibrillator and cardiac resynchronization devices. Eur Heart J. 2015:1-9.

27. Healey JS, Lopes RD, Connolly SJ. The detection and treatment of subclinical atrial fibrillation: evaluating the IMPACT of a comprehensive strategy based on remote arrhythmia monitoring. Eur Heart J. 2015:102.

28. Ringleb PA, Bousser MG, Ford G, Bath P, Brainin M, Caso V, et al. Guidelines for management of ischaemic stroke and transient ischaemic attack 2008. Cerebrovasc Dis. 2008;25:457-507.
29. Goldstein LB, Bushnell CD, Adams RJ, Appel LJ, Braun LT, Chaturvedi $S$, et al. Guidelines for the Primary Prevention of Stroke: A Guideline for Healthcare Professionals From the American Heart Association/ American Stroke Association. Stroke. 2014;45:3754-832.

30. Granger CB, Alexander JH, McMurray JJ, Lopes RD, Hylek EM, Hanna $\mathrm{M}$, et al. Apixaban versus warfarin in patients with atrial fibrillation. $\mathrm{N}$ Engl J Med. 2011;365:981-92.

31. Connolly SJ, Ezekowitz MD, Yusuf S, Eikelboom J, Oldgren J, Parekh A et al. Dabigatran versus warfarin in patients with atrial fibrillation. $N$ Engl J Med. 2009;361:1139-51.

32. Giugliano RP, Ruff CT, Braunwald E, Murphy SA, Wiviott SD, Halperin $\mathrm{JL}$, et al. Edoxaban versus warfarin in patients with atrial fibrillation. $\mathrm{N}$ Engl J Med. 2013;369:2093-104.

33. Patel MR, Mahaffey KW, Garg J, Pan G, Singer DE, Hacke W, et al Rivaroxaban versus warfarin in nonvalvular atrial fibrillation. $N$ Engl $J$ Med. 2011;365:883-91. 


\section{Duração dos Episódios de Fibrilhação Auricular e Implicações no Risco Tromboembólico}

Acta Med Port 2015:28:766-772

Publicado pela Acta Médica Portuguesa, a Revista Científica da Ordem dos Médicos

Av. Almirante Gago Coutinho, 151

1749-084 Lisboa, Portugal.

Tel: +351218428215

E-mail: submissao@actamedicaportuguesa.com

www.actamedicaportuguesa.com

ISSN:0870-399X | e-ISSN: 1646-0758

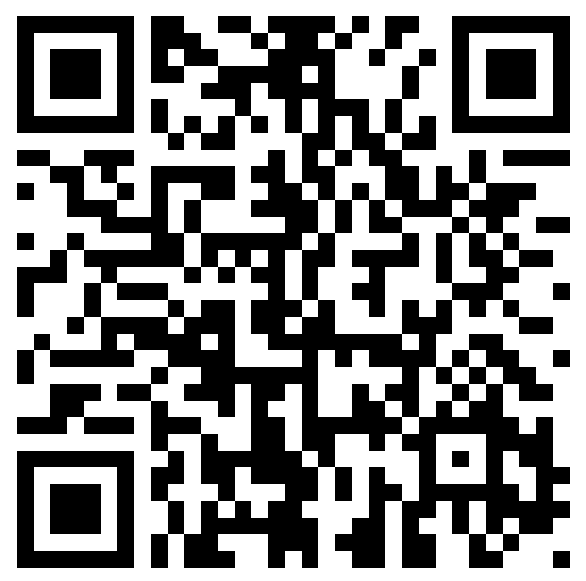

